\title{
Minimally invasive approach for the treatment of intradural spinal pathology
}

\author{
Ravi H. Gandhi, M.D., and John W. German, M.D. \\ Division of Neurosurgery, Albany Medical Center, Albany, New York
}

\begin{abstract}
Object. A wide variety of spinal intradural pathology traditionally has been treated from a midline posterior laminectomy using standard microsurgical techniques. This approach has been successful in treating the pathology; however, it carries a risk of postoperative complications including CSF leakage, wound infection, and spinal instability. The authors describe a minimally invasive surgical (MIS) approach to treating spinal intradural pathology with a low rate of postoperative complications.

Methods. Through a retrospective review of a prospectively collected surgical database, the authors identified 26 patients who underwent 27 surgeries via an MIS approach for intradural pathology of the spine. Using a tubular retractor system and an operative microscope, the authors were able to treat all patients with a unilateral, paramedian, and muscle-splitting technique. They then collected data regarding operative blood loss, length of stay, imaging characteristics, and outcomes.

Results. Eight cervical, 8 thoracic, and 11 lumbar intradural pathological entities, which included 14 oncological lesions, 4 Chiari I malformations, 4 arachnoid cysts, 3 tethered cords, 1 syrinx, and 1 chronic visceral pain, were treated via an MIS approach. The average blood loss was $197 \mathrm{ml}$ and the average hospital stay was 3 days. One patient had to return to the operating room for noninfectious wound dehiscence. One patient required reoperation 18 months after the initial surgery for recurrence of the initial pathology. There was no CSF leak, no infection, and no spinal instability associated with the initial surgery on follow-up.

Conclusions. Intradural spinal pathology can be safely and effectively treated with MIS approaches without an increased risk of neurological injury. This approach may also offer a reduced postoperative length of stay, risk of CSF leak, and risk of future spinal instability.
\end{abstract}

(http://thejns.org/doi/abs/10.3171/2013.5.FOCUS13163)

KEY WORDS $\bullet$ minimally invasive $\bullet$ intradural $\bullet$ meningioma $\bullet$
hemangioblastoma $\bullet$ Chiari malformation $\bullet$ tethered cord $\bullet$ arachnoid cyst

I NTRADURAL pathology of the spine consists of oncological and nononcological lesions. Either pathology can be divided into intramedullary and extramedullary lesions. The most common intramedullary pathology consists largely of glial tumors, but other neoplasms such as hemangioblastomas, metastatic lesions, and benign pathological processes can be seen. Extramedullary, intradural pathology includes neoplasms (nerve sheath tumors and meningiomas) as well as nononcological lesions such as arachnoid cysts, tethered cord, and syringomyelia.

Clinical presentation can vary widely, ranging from axial or radicular pain to neurological deficits. It is the responsibility of the clinician to elucidate the correlation between imaging findings and symptoms. Advanced imaging techniques such as MRI and CT myelography are cal.

Abbreviation used in this paper: $\mathrm{MIS}=$ minimally invasive surgi- invaluable tools in the assessment of these patients and in planning the best management approach.

A midline incision with bilateral muscle dissection, removal of the posterior elements, and wide exposure of the dorsal spinal cord remains the mainstay of treatment. This approach has afforded surgeons access for successful removal of intradural pathology, but there is a risk of CSF leakage, spinal deformity, and postoperative pain. This approach has been modified with hemilaminectomy and partial facetectomy to reduce the risk of postoperative pain and subsequent spinal deformity. ${ }^{2,7,13}$

The use of MIS approaches has been well described for the treatment of degenerative spinal disease. These techniques are now being adopted and modified for the treatment of intradural pathology. ${ }^{4,12}$ We elaborate on our experience with MIS approaches in the treatment of various types of intradural pathology and suggest that this technique may contribute to faster postoperative recovery, a lower CSF leakage rate, and a decrease in the risk 


\section{R. H. Gandhi and J. W. German}

of spinal instability, by reducing the degree of musculoligamentous disruption.

\section{Methods}

Through a retrospective review of a prospectively collected surgical database, we identified 26 patients who underwent 27 surgeries via an MIS approach for intradural pathology of the spine. All patients underwent a physical examination and findings were correlated with preoperative imaging. Institutional review board approval was obtained from Albany Medical College.

Details regarding the operation, including duration, estimated blood loss, and need for transfusion were prospectively recorded. Postoperative images were obtained and reviewed when appropriate based on pathology. Information about operative and postoperative complications including CSF leakage, readmission, return to the operating room, infection, and increased neurological deficit was also recorded and analyzed.

In the thoracolumbar spine, a K-wire was used to localize the correct level on fluoroscopy. After infiltrating the skin with lidocaine, a 2- to $2.5-\mathrm{cm}$ paramedian incision was created, and an expandable tubular retractor (Quadrant Retractor, Medtronic Sofamor-Danek) was placed over sequential dilators using a muscle-splitting technique. In the cervical spine, a midline incision was created and a lateral fascial incision was made. Next a muscle-splitting dissection was carried out down to the lamina and sequential dilators were placed. The bone removal was carried out with the use of high-speed drills and Kerrison rongeurs. Once the dura was incised and the leaflets were tacked up, the intradural pathology was treated using a microsurgical technique. The dura was closed primarily or using a dural substitute. The dural suture line was then covered with a dural sealant.

All patients were then mobilized within 24 hours after surgery. Postoperative imaging was performed when indicated based on the pathology.

\section{Illustrative Cases}

Case 1

A 46-year-old woman presented with progressive hemisensory changes and gait difficulty. Follow-up MRI with contrast demonstrated progressive growth of a foramen magnum- $\mathrm{C} 1$ mass with compression of the cervicomedullary junction (Fig. 1). The patient underwent an MIS approach with partial occipital craniotomy, C-1 and C-2 hemilaminotomy, and resection of the intradural mass, which was confirmed to be a meningioma (Fig. 2). There was a 50-ml blood loss. A watertight dural closure was performed, and the patient was discharged to home on postoperative Day 3. Postoperative imaging demonstrated complete resection of the mass and no evidence of a pseudomeningocele (Fig. 3). The patient had complete resolution of her symptoms.

\section{Case 2}

A 59-year-old man presented with back and bilateral
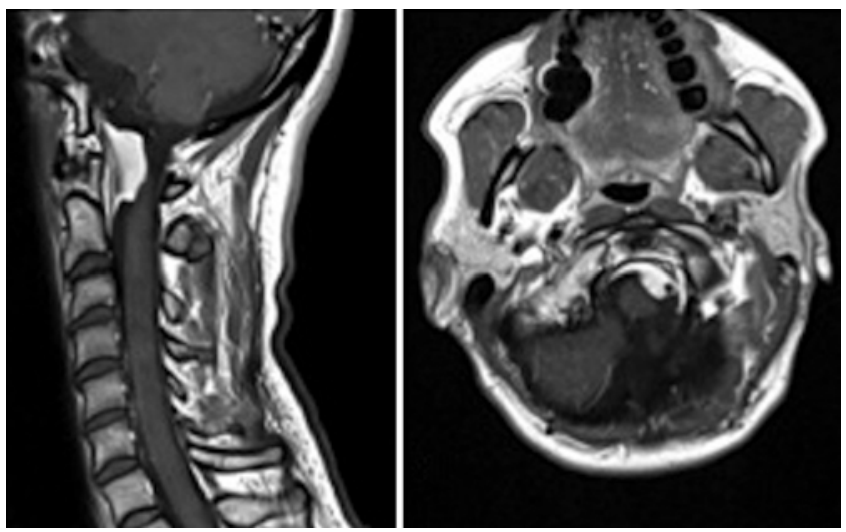

FIG. 1. Case 1. Preoperative MR images. T1-weighted sagittal (left) and axial (right) Gd-enhanced MR images demonstrating a lesion ventral and lateral to the spinal cord at C-1.

leg pain. Magnetic resonance imaging showed a large enhancing mass at the L3-4 interspace with multiple flow voids (Fig. 4A and B). The patient underwent a preoperative catheter angiography that was negative for a vascular abnormality. An L3-4 laminotomy was performed via an MIS approach. The estimated blood loss was 400 $\mathrm{ml}$; however, the patient did not require a blood transfusion. The patient was discharged on postoperative Day 1. Postoperative MRI did not show evidence of recurrence or pseudomeningocele (Fig. 4C and D).

\section{Case 3}

A 54-year-old woman presented with tussive headaches and paresthesia in her hands. An MR image showed a Chiari I malformation with a syrinx that exhibited progression on subsequent imaging (Fig. 5 left). The patient subsequently underwent an MIS approach for a suboccipital decompression with removal of C-1 and duraplasty. Following the operation, the patient's headache improved and follow-up imaging showed the syrinx had resolved (Fig. 5 right).

\section{Case 4}

A 34-year-old man presented with progressive my-

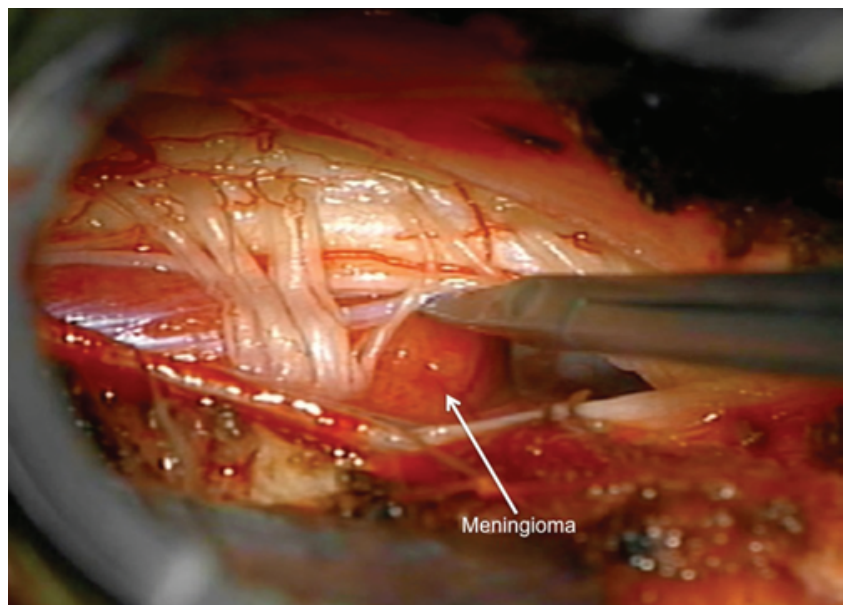

FIG. 2. Case 1. Intraoperative photograph demonstrating a meningioma (arrow) ventral to the $\mathrm{C}-1$ nerve roots. 

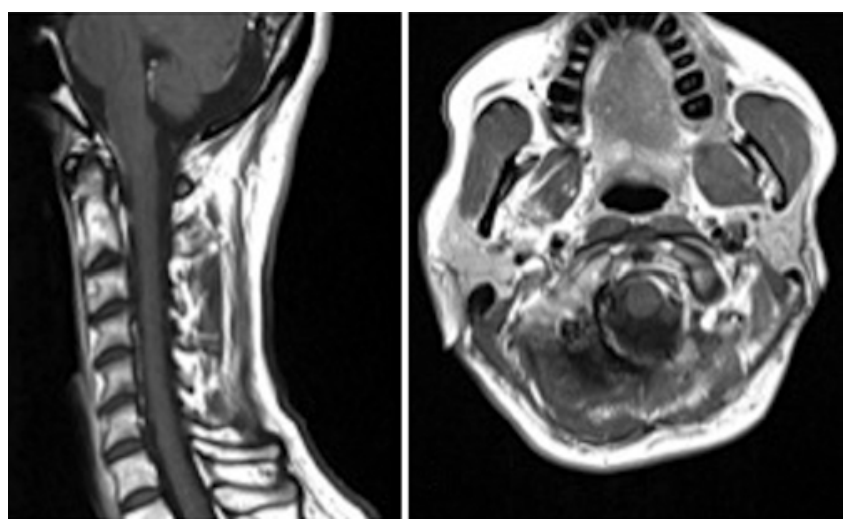

Fig. 3. Case 1. Postoperative T1-weighted sagittal (left) and axial (right) Gd-enhanced MR images demonstrating a complete resection of a foramen magnum-C1 meningioma.

elopathy with severe difficulty with gait and manual dexterity that began after a stretching episode. The patient was found to have a syringomyelia from C-6 to T-1 with no other underlying abnormality (Fig. 6 left). He underwent a fenestration and subarachnoid shunting of the syrinx via an MIS approach to a C-7 hemilaminectomy. The patient's symptoms resolved except for minimal residual neck pain, and postoperative imaging demonstrated significant improvement in the size of the syrinx (Fig. 6 right).

\section{Results}

Of the 26 patients who underwent surgery for intradural spinal pathology via an MIS approach, 14 were female and 12 were male. Ten of 26 patients presented with a chief complaint of pain. The remaining 16 patients presented with neurological deficits including 6 patients with
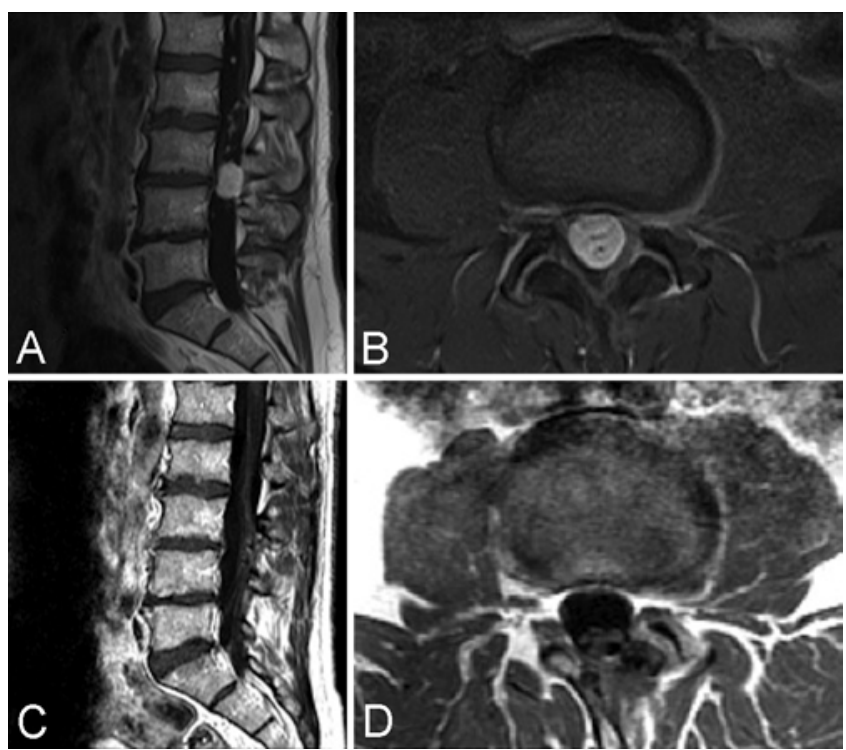

FIG. 4. Case 2. Preoperative and postoperative MR images. T1weighted sagittal (A) and axial (B) Gd-enhanced MR images demonstrating a lesion at L3-4 interspace. T1-weighted sagittal (C) and axial (D) Gd-enhanced MR images demonstrating a complete resection of an L3-4 hemangioblastoma.
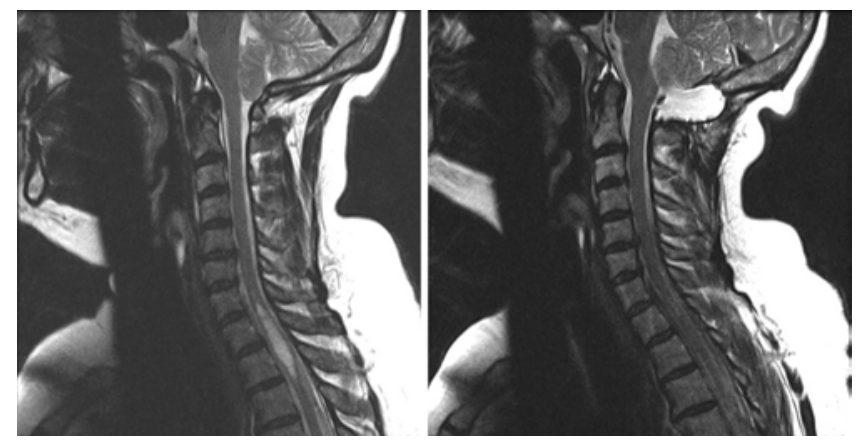

FIG. 5. Case 3. Left: Preoperative T2-weighted image demonstrating a Chiari I malformation with a syrinx at C7-T3. Right: Postoperative T2-weighted image demonstrating a Chiari decompression with resolution of the syrinx.

myelopathy, 3 with urinary incontinence, 1 with paraplegia, 3 with gait instability, 1 with hand weakness, and 2 with paresthesia (Table 1).

A total of 14 intradural tumors were treated, of which 12 were extramedullary and 2 were intramedullary. There were 4 cervical, 6 thoracic, and 4 lumbar tumors. There were 4 meningiomas, 4 nerve sheath tumors, 2 hemangioblastomas, 1 myxopapillary ependymoma, 1 metastatic carcinoma, 1 neuroendocrine tumor, and 1 pseudotumor. Eleven patients had a complete resection confirmed during the operation and on postoperative imaging. The patient with the neuroendocrine tumor underwent a complete resection at the level treated; however, he had multiple metastatic lesions with diffuse dural enhancement. Two patients underwent a biopsy for diagnosis as well as decompression. The remaining conditions treated included 4 Chiari decompressions, 4 arachnoid cysts, 3 tethered cords, 1 syringosubarachnoid shunt, and 1 midline myelotomy and cordotomy for chronic visceral pain from cancer.

The mean intraoperative blood loss was $197 \mathrm{ml}$ (range 15-900 ml). Only 1 patient required a blood transfusion, and this was related to anemia from chronic disease due to her cancer rather than anemia from the surgery. One patient had a fusion at the time of surgery due to complete removal of the ipsilateral facet. Another patient required subsequent fusion at the level above the surgery. This patient was symptomatic from degenerative disc disease and had already had spinal surgery at other levels in the
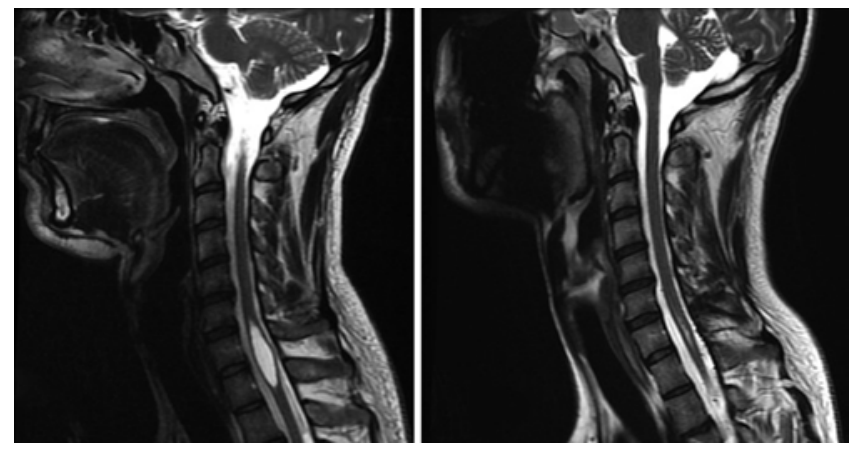

FIG. 6. Case 4. Left: Preoperative T2-weighted image demonstrating a thoracic syringomyelia. Right: Postoperative T2-weighted image demonstrating a syringomyelia-arachnoid shunt. 
R. H. Gandhi and J. W. German

TABLE 1: Preoperative and postoperative findings

\begin{tabular}{|c|c|c|}
\hline Case No. & Preop Symptoms & Postop Outcome \\
\hline 1 & back pain \& hamstring tightness after minor trauma & stable \\
\hline 2 & myelopathy & stable \\
\hline 3 & C-8 radiculopathy & complete resolution \\
\hline 4 & back pain \& paresthesia & complete resolution \\
\hline 5 & back pain \& hamstring pain \& tightness & minor hypesthesia \\
\hline 6 & paresthesia \& gait instability & complete resolution \\
\hline 7 & myelopathy & resolution of hand weakness \& improvement in leg weakness \\
\hline 8 & paresthesia, gait instability, \& headache & improved headache \& instability \\
\hline 9 & chronic visceral pain & decreased drug dependence; discharged home; death \\
\hline 10 & bilat lower-extremity radiculopathy & complete resolution \\
\hline 11 & myelopathy & stable \\
\hline 12 & myelopathy & improved \\
\hline 13 & paraplegia & death \\
\hline 14 & S-1 radiculopathy & stable \\
\hline 15 & back \& leg pain & improved \\
\hline 16 & bilat claudication & improved \\
\hline 17 & It hemisensory loss \& occipital neuralgia & minimal residual paresthesia \\
\hline 18 & back pain & improved \\
\hline 19 & gait instability \& bladder dysfunction & improved \\
\hline 20 & myelopathy \& neck pain & improved \\
\hline 21 & myelopathy & stable \\
\hline 22 & back \& leg pain & stable \\
\hline 23 & bladder dysfunction & stable \\
\hline 24 & gait instability \& headache & improved \\
\hline 25 & myelopathy & improved \\
\hline 26 & headache & improved \\
\hline 27 & leg pain \& incontinence & stable \\
\hline
\end{tabular}

past, suggesting that the need for fusion was unrelated to the tumor resection surgery. There were no conversions to open traditional surgery and no postoperative CSF leaks. Seventy-five percent of Chiari decompression patients had pseudomeningoceles that were seen on postoperative imaging, but none were symptomatic. Two patients died within 90 days of surgery, but both deaths were related to the patients' primary disease and not to the surgery itself. One patient was readmitted with aseptic meningitis after Chiari decompression and another patient was readmitted for abdominal pain and nausea. Neither of those patients required any further surgical treatment. One patient required a return to the operating room 2 weeks after surgery for wound dehiscence; however, there was no infection or CSF leakage noted at that time. One patient required repeat surgery for further fenestration of a large arachnoid cyst. The cyst had multiple septations; therefore the second surgery addressed another compartment of the cyst at another level (Table 2).

All patients were mobilized and ambulatory within 24 hours of surgery except for 1 patient who underwent a midline myelotomy and cordotomy for intractable visceral pain and another with metastatic carcinoma. The mean length of stay in the hospital was 3 days; 22 patients were discharged home, 4 went to rehabilitation, and 1 died. At the time of the writing of this paper, 1 patient had increased neuropathic pain after surgery, 7 patients $(26 \%)$ had complete resolution of their chief complaint, 10 patients $(37 \%)$ improved with minimal residual problems, and 7 patients $(26 \%)$ remained stable. One patient died of cancer-related complications. Another patient with intractable visceral pain from cancer requiring long-term intravenous medication drips was able to be weaned off the medication and discharged to home after a midline myelotomy and cordotomy.

\section{Discussion}

Since Foley and $\mathrm{Smith}^{3}$ popularized the microendoscopic technique, there has been a wealth of literature addressing the utility and potential benefit of that technique. Experience with tubular retractor systems and MIS technique has allowed surgeons to expand the application of these techniques to treat various spinal pathologies. The technique has been well described for the purposes of decompression, stabilization, and various extradural pathologies. This series further adds to the literature showing that MIS techniques can prove useful for all intradural 
Minimally invasive surgery for spinal pathology

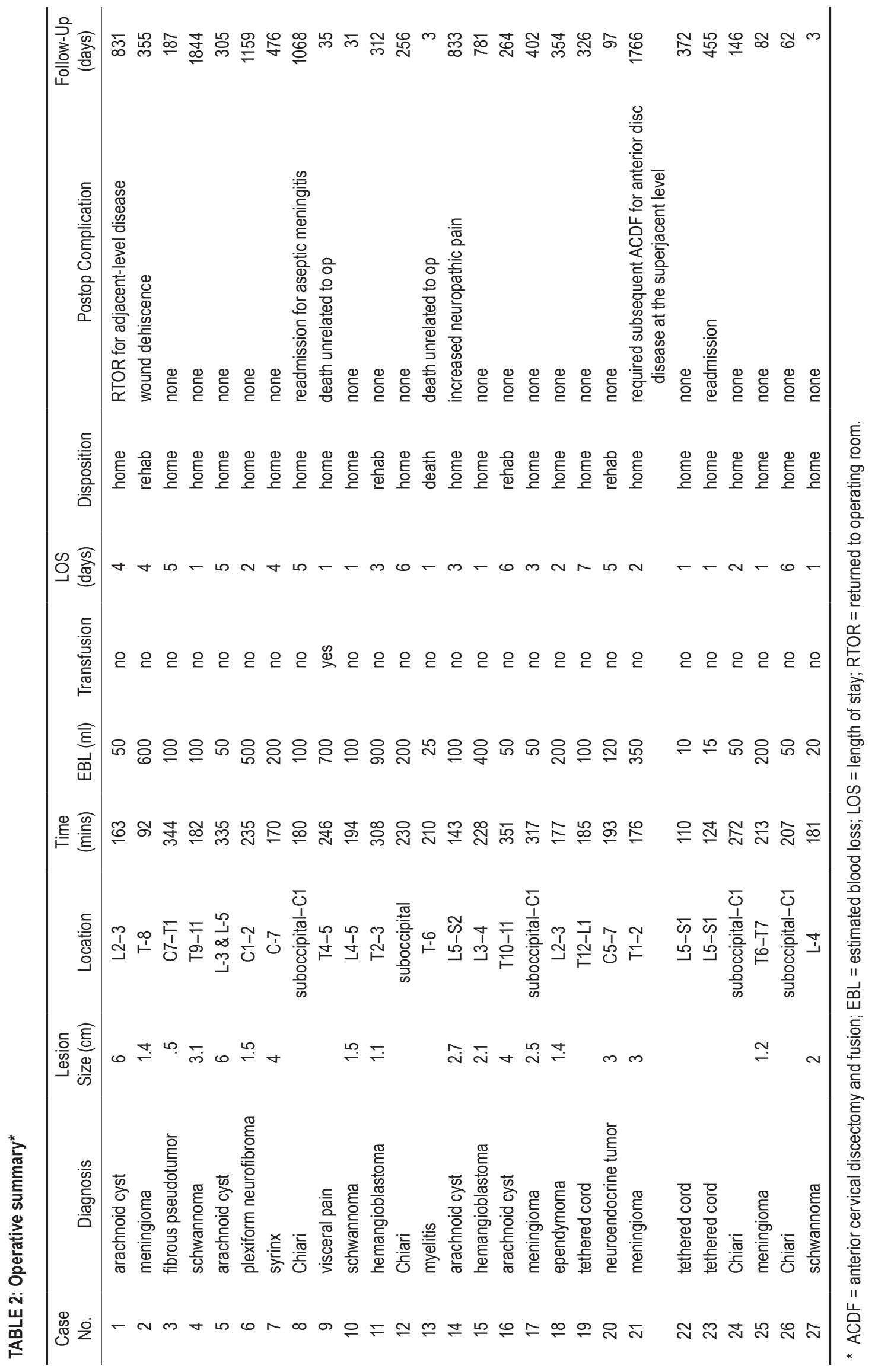




\section{R. H. Gandhi and J. W. German}

pathology, intra- and extramedullary pathology as well as spinal dysraphism, syringomyelia, and treatment of Chiari malformation.

A traditional approach to intradural pathology of the spine involves a midline incision, bilateral muscle dissection, and removal of the posterior spinal elements. A rate of instability from this approach requiring a second operation has been reported as high as 60\%..$^{5}$ In a series by Seppälä et al. looking at schwannoma resection in Finland, there was a $10 \%$ postoperative complication rate and a $6 \%$ delayed spinal instability rate. ${ }^{9}$ There have been improvements in open technique using a hemilaminotomy approach. This approach has been associated with fewer complications, better outcomes, and shorter hospital stays..$^{2,7,10,13,14}$ In our series, only 1 patient underwent fusion at the time of surgery (Case 3 ) because the entire facet was removed to reach the tumor. One patient required anterior cervical discectomy and fusion for degenerative disc disease, but this was to treat a preexisting condition. Although dynamic imaging was not customarily used in our series, no other patients required subsequent surgery for the treatment of instability or symptomatic kyphosis.

In an attempt to further advance minimally invasive approaches to the spine, tubular retractors with musclesplitting techniques have been applied to the treatment of intradural pathology. This minimizes muscle destruction and affords an adequate corridor to tumor resection. There is also a decrease in the dead space at the end of surgery, theoretically limiting the possibility of postoperative CSF leakage. By minimizing tissue destruction and unnecessary bone removal, the risk of postoperative instability is decreased. Haji et al. ${ }^{4}$ have described the use of a minimally invasive technique to remove both intra- and extradural neoplasms including 2 intramedullary lesions without added neurological risk. Treway et al. ${ }^{11}$ presented a series of 3 patients in whom they used a minimally invasive approach for the treatment of tethered cord syndrome, with reduced blood loss and length of hospital stay.

Our series further demonstrates that a wide variety of intradural pathologies can be treated with an MIS approach. Of the 14 oncological lesions that were treated, 12 were completely resected. The 2 tumors that were not completely resected would have been incomplete using any surgical approach. None of the tumors have recurred. Of the 4 patients who underwent suboccipital craniectomy and dural augmentation for Chiari I malformation, all had an improvement in their headaches and gait instability. Although postoperative imaging showed a $75 \%$ rate of pseudomeningocele, higher than the 5\% reported in the literature, there was no need for reoperation in our series. Of note in these cases is that the dura was loosely reconstructed using a dural substitute (Duragen, Integra Life Sciences) and a few tack-up sutures. Three patients underwent surgical fenestration of arachnoid cysts. One patient had to return to the operating room 18 months later for a repeat fenestration at another level. Although the rate of reoperation for arachnoid cyst was higher than expected, the need for reoperation was not a result of limitations of the exposure, but rather due to an underestimation as to the extent of the arachnoid cyst septations on preoperative imaging. The 3 patients who underwent spinal cord untethering did not have any complications from surgery: 2 patients remained stable from a chief complaint standpoint and 1 patient had a significant improvement in his incontinence 6 weeks after surgery. One patient had a significant decrease in the size of the syrinx after an MIS approach to syringomyelia-arachnoid shunt placement with significant improvement in his myelopathic symptoms. One patient had a punctate midline myelotomy and cordotomy for the treatment of chronic visceral pain. Although there was no change in her functional status due to her cancer, she was able to leave the hospital with a significant reduction in her requirement for continuous pain medications.

Although the average length of hospital stay of 3 days in this series is comparable to that of series of open resection, ${ }^{2,10,13}$ the heterogeneity and characteristics of our population may have artificially lengthened that average. Twenty-five patients were ambulating within 24 hours of surgery and $48 \%$ of patients were discharged to home by postoperative Day 2. In our anecdotal experience and in other series,${ }^{10}$ patients undergoing an open excision are often kept on bed rest for 24-72 hours, thus increasing the risk of pressure ulcers and deep vein thrombosis. Although our average blood loss of $197 \mathrm{ml}$ (range 15-900 $\mathrm{ml}$ ) is higher than other series, ${ }^{4,6}$ only 1 patient in our series required transfusion. This may represent our learning curve.

We have demonstrated that this technique can be applied to a wide array of intradural pathologies; however there are added complexities that must be addressed. To deal with pathology at the midline or the spinal cord, a pedicle-to-pedicle exposure is preferred. To facilitate obtaining this exposure, we add resection of the ventral portion of the spinous process to maintain a medial trajectory. It remains important to carefully review the preoperative images to appropriately plan the levels that need to be approached and a trajectory that best suits the pathology. Although a smaller postoperative dead space may help reduce CSF leakage, we believe that a watertight dural closure is often preferable. One exception is following treatment of a Chiari malformation where our preference has been to keep the dura loosely approximated to optimize decompression. Dural closure can be difficult with a smaller working space. Park et al. ${ }^{8}$ have described dural closure with a U-clip (Medtronic). In our experience, the 4.0 Vicryl stitch on a U-shaped needle has been adequate. Meticulous muscle and fascial closure are also important in preventing CSF leak.

Although we've shown that there is a wide application of an MIS approach, there are limitations to its use. Lesions extending more than 2 spinal segments may not be best treated via an MIS approach, as the expandable tubular retractor is often limited. Changing the angle of the retractor is feasible; however, doing so may increase the risk of surgery. Pathology with a large ventral component may not be best addressed by an MIS approach. This series demonstrates our experience with the application of MIS approaches to intradural pathology, but there is a significant learning curve when adopting such an approach. The senior author (J.W.G.) has had a robust experience 
with minimally invasive surgery for other spinal pathology, and the transition to applying these techniques for intradural pathology was gradual. There does not seem to be a significant increase in operative time, but this is anecdotal, and considering our heterogeneous group it is difficult to compare. There was also no improvement in our operative time with added experience (the cases in Table 2 are organized in chronological order). After obtaining experience with MIS approaches, the combination of those techniques with traditional microsurgical techniques is natural. It is our recommendation that surgeons should have adequate experience with MIS approaches prior to attempting resection of intradural pathology with this method.

\section{Conclusions}

We have demonstrated that MIS approaches can be used to treat a large array of intradural pathology of both the intramedullary and extramedullary compartments. We have shown that neoplasms can be effectively and safely resected with a lower rate of CSF leakage and postoperative complications. We have confirmed earlier reports that resection of intradural, extramedullary neoplastic lesions, ${ }^{4,12}$ intramedullary, ${ }^{4,6}$ and cord detethering ${ }^{11}$ can be accomplished. We have also described the use of MIS approaches to perform a Chiari decompression including intradural exploration and tonsillar resection, to perform a midline punctate myelotomy and cordotomy for intractable cancer related pain, for an intramedullary biopsy, and for syringosubarachnoid shunting. The use of a tubular retractor system and muscle splitting techniques allow for adequate visualization and a decrease in anatomical injury. This should allow for less postoperative pain and risk of spinal instability; however, this will require more extensive randomized, prospective trials to elucidate.

\section{Disclosure}

The authors report no conflict of interest concerning the materials or methods used in this study or the findings specified in this paper.

Author contributions to the study and manuscript preparation include the following. Conception and design: Gandhi. Acquisition of data: German. Analysis and interpretation of data: Gandhi. Drafting the article: both authors. Critically revising the article: German. Reviewed submitted version of manuscript: both authors. Administrative/technical/material support: German. Study supervision: German.

\section{References}

1. Batzdorf U, McArthur DL, Bentson JR: Surgical treatment of
Chiari malformation with and without syringomyelia: experience with 177 adult patients. Clinical article. J Neurosurg 118:232-242, 2013

2. Chiou SM, Eggert HR, Laborde G, Seeger W: Microsurgical unilateral approaches for spinal tumour surgery: eight years' experience in 256 primary operated patients. Acta Neurochir (Wien) 100:127-133, 1989

3. Foley KT, Smith MM, Rampersaud YR: Microendoscopic approach to far-lateral lumbar disc herniation. Neurosurg Focus 7(5):e5, 1999

4. Haji FA, Cenic A, Crevier L, Murty N, Reddy K: Minimally invasive approach for the resection of spinal neoplasm. Spine (Phila Pa 1976) 36:E1018-E1026, 2011

5. Ogden AT, Bresnahan L, Smith JS, Natarajan R, Fessler RG: Biomechanical comparison of traditional and minimally invasive intradural tumor exposures using finite element analysis. Clin Biomech (Bristol, Avon) 24:143-147, 2009

6. Ogden AT, Fessler RG: Minimally invasive resection of intramedullary ependymoma: case report. Neurosurgery 65:E1203E1204, 2009

7. Oktem IS, Akdemir H, Kurtsoy A, Koç RK, Menkü A, Tucer B: Hemilaminectomy for the removal of the spinal lesions. Spinal Cord 38:92-96, 2000

8. Park P, Leveque JC, La Marca F, Sullivan SE: Dural closure using the U-clip in minimally invasive spinal tumor resection. J Spinal Disord Tech 23:486-489, 2010

9. Seppälä MT, Haltia MJ, Sankila RJ, Jääskeläinen JE, Heiskanen O: Long-term outcome after removal of spinal schwannoma: a clinicopathological study of 187 cases. J Neurosurg 83:621-626, 1995

10. Sridhar K, Ramamurthi R, Vasudevan MC, Ramamurthi B: Limited unilateral approach for extramedullary spinal tumours. Br J Neurosurg 12:430-433, 1998

11. Tredway TL, Musleh W, Christie SD, Khavkin Y, Fessler RG, Curry DJ: A novel minimally invasive technique for spinal cord untethering. Neurosurgery 60 (2 Suppl 1):ONS70-ONS74, 2007

12. Tredway TL, Santiago P, Hrubes MR, Song JK, Christie SD, Fessler RG: Minimally invasive resection of intradural-extramedullary spinal neoplasms. Neurosurgery 58 (1 Suppl): ONS52-ONS58, 2006

13. Yaşargil MG, Tranmer BI, Adamson TE, Roth P: Unilateral partial hemi-laminectomy for the removal of extra- and intramedullary tumours and AVMs. Adv Tech Stand Neurosurg 18:113-132, 1991

14. Yu Y, Zhang X, Hu F, Xie T, Gu Y: Minimally invasive microsurgical treatment of cervical intraspinal extramedullary tumors. J Clin Neurosci 18:1168-1173, 2011

Manuscript submitted April 14, 2013.

Accepted May 24, 2013.

Please include this information when citing this paper: DOI: 10.3171/2013.5.FOCUS13163.

Address correspondence to: Ravi H. Gandhi, M.D., Division of Neurosurgery, Albany Medical Center, 43 New Scotland Ave., Mail Code 10, Albany, NY 12208. email: rhgandhi@gmail.com. 